\title{
A simple 2-D interpolation model for analysis of nonlinear data
}

\author{
Mehdi Zamani
}

Department of Civil Engineering, Faculty of Technology and Engineering, Yasouj University, Yasouj, Iran; mahdi@mail.yu.ac.ir

Received 1 March 2010; revised 24 April 2010; accepted 13 May 2010.

\begin{abstract}
To determination the volume and weight of nonuniform bodies, such as in ore deposits evaluation for mining and rock cutting for construction, the methods of interpolation are usually used. The classic curves, which are frequently used to interpolate one-dimensional data are cubic spline, Bspline and Bezier curves. These methods have good efficiency for determination of geometric characteristics of nonregular masses. They have some limitations and problems with twodimensional interpolation analysis such as forming large linear systems of equations with a lot of entries and difficulty encounter with their solutions. In this research the two-dimensional splines are used, which have the advantages of simplicity and less computational operations effort. The spline functions that are applied have the continuity of $C^{1}$ at elements boundaries. The presented model has suitable efficiency for volumes of large extents governing to lots of data.
\end{abstract}

Keywords: Simulation; Approximation; Least square; Cubic Spline; Optimization; Curve Fitting; Prediction

\section{INTRODUCTION}

The most popular methods for interpolation of data are Lagrange method, Neville iteration approach, Newton divided difference methods, Cubic spline, Hermit spline, Bspline and Bezier methods. For the Neville and Newton divided difference method, the higher degree of polynomial generation is straightforward. In conic spline approach each curve consists of a number of about $n$ segment curves. The general way is to divide the interval to collect subintervals or segments and construct different approximating polynomial on each interval. Approximation by functions of this type is called piecewise polynomial approximation. This method has the advantage of removing the oscillatory nature of high degree polynomials. In this approach there exists the continuity of $C^{2}$ on the segments boundaries. However; the continuity order is optional to users willing. The formulation for creating cubic spline curve results in the solution of a three-diagonal system of equations. Hermit interpolation are based on two points $p_{1}$ and $p_{2}$ and two tangent values $p_{1}^{\prime}$ and $p_{2}^{\prime}$ at those points. It computes a curve segment that starts at $p_{1}$, going in direction to $p_{1}^{\prime}$ and ends at $p_{2}$ moving toward $p_{2}^{\prime}$. Hermit interpolation has an important advantage. The Hermit curve can be modified by changing the tangent values.

The Bezier curve is a parametric curve $p(t)$ that is a polynomial function of the parameter $t$. The degree of the polynomial depends on the number of points used to define the curve. The method applies control points and presents an approximating curve. The Bezier curve does not pass through internal points but the first and last points. Internal points influence the direction and position of the curve by pulling it toward themselves [1-3]. B-spline methods were first proposed in the 1940 for curves and surfaces. The B-spline curves can be approximating or interpolating curves. The advantage of B-spline curves to Bezier curve is the obtaining the higher continuity for the individual spline segments [2-5]. If there are a set of triple data $c=\left\{\left(x_{i}, y_{i}, y_{i}^{\prime}\right)\right.$, $i=0,1,2, \ldots, n\}$ The cubic spline functions $S_{i}(x)$ can be obtained on each interval $\left[x_{i}, x_{i+1}\right]$. With this model the continuity of $C^{1}$ exists on the boundary of each segment. The spline function of degree 3 for each interval is:

$$
g_{i}(x)=a_{i}+b_{i} x+c_{i} x^{2}+d_{i} x^{3}=\left[\begin{array}{llll}
1 & x & x^{2} & x^{3}
\end{array}\right]\left[\begin{array}{c}
a_{i} \\
b_{i} \\
c_{i} \\
d_{i}
\end{array}\right]
$$

If the Eq.1 is written based on the first point of each segment $\left(x_{i}, x_{i+1}\right)$ then, 


$$
\begin{gathered}
s_{i}(x)=a_{i}+b_{i}\left(x-x_{i}\right)+c_{i}\left(x-x_{i}\right)^{2}+d_{i}\left(x-x_{i}\right)^{3}= \\
{\left[\begin{array}{llll}
1 & x & x^{2} & x^{3}
\end{array}\right]\left[\begin{array}{cccc}
1 & -x_{i} & x_{i}^{2} & x_{i}^{3} \\
0 & 1 & -2 x_{i} & 3 x_{i}^{2} \\
0 & 0 & 1 & -3 x_{i}^{2} \\
0 & 0 & 0 & 1
\end{array}\right]\left[\begin{array}{l}
a_{i} \\
b_{i} \\
c_{i} \\
d_{i}
\end{array}\right]}
\end{gathered}
$$

The first derivatives of the spline function (2) is as follows,

$$
\begin{aligned}
& s_{i}^{\prime}(x)=b_{i}+2 c_{i}\left(x-x_{i}\right)+3 d_{i}\left(x-x_{i}\right)^{2}= \\
& {\left[\begin{array}{lll}
1 & x & x^{2}
\end{array}\right]\left[\begin{array}{ccc}
1 & -2 x_{i} & 3 x_{i}^{2} \\
0 & 2 & -6 x_{i} \\
0 & 0 & 3
\end{array}\right]\left[\begin{array}{l}
b_{i} \\
c_{i} \\
d_{i}
\end{array}\right]}
\end{aligned}
$$

The coefficients $a_{i}, b_{i}, c_{i}$ and $d_{i}$ for each interval can be obtained explicitly from the Eq.5.

$$
\left[\begin{array}{c}
a_{i}=s\left(x_{i}\right)=f_{i} \\
b_{i}=s^{\prime}\left(x_{i}\right)=f_{i}^{\prime} \\
c_{i}=\frac{3}{h_{i}^{2}}\left(f_{i+1}-f_{i}\right)-\frac{1}{h_{i}}\left(f_{i+1}^{\prime}+2 f_{i}^{\prime}\right), \\
d_{i}=-\frac{2}{h_{i}^{3}}\left(f_{i+1}-f_{i}\right)+\frac{1}{h_{i}^{2}}\left(f_{i+1}^{\prime}+f_{i}^{\prime}\right)
\end{array}\right.
$$

where $h_{i}$ is the element size or the element interval $\left(h_{i}=\right.$ $\left.x_{i+1}-x_{i}\right)$. The problem with this approach is that we generally don't have the tangent values at $n+1$ point. This can be provided by applying the following approximation equations (forward difference, central difference and backward difference approximation, respectively) for the first derivatives at the points and for uniform intervals $h_{i}$.

$$
\left[\begin{array}{l}
f_{i}^{\prime}=\frac{1}{2 h_{i}}[-3,4,1] \\
f_{i}^{\prime}=\frac{1}{2 h_{i}}[-1,0,1] \\
f_{i}^{\prime}=\frac{1}{2 h_{i}}[1,-4,3]
\end{array}\right.
$$

The following two examples show the interpolation of two sets of data by the above approach.

\section{EXAMPLES}

\subsection{Example 1}

This example is in the field of groundwater engineering. When a production well in unconfined aquifer is pumped, water is continuously withdrawn from storage within the aquifer as the cone of depression progresses radially outward from the well. Because of the absence of a re- charge source in the form of vertical leakage, there can be no stabilization of water levels and the head $(h)$ in the aquifer will continue to decline supposing the aquifer is infinite in areal extent. However, the rate of decline of head $(h)$ continuously decreases as the cone of depression spreads. The partial differential equation governing the unsteady-state radial flow in the nonleaky confined aquifer in polar coordinate system is,

$$
\frac{\partial^{2} h}{\partial r^{2}}+\frac{1}{r} \frac{\partial h}{\partial r}=\frac{S}{T} \frac{\partial h}{\partial t} \text {. }
$$

The Theis solution for the above partial differential equation is,

$$
s=h_{0}-h=\frac{Q}{4 \pi T} \int_{u}^{\infty} \frac{e^{-u}}{u} d u .
$$

Theis approximated the above equation by the following series,

$$
h_{0}-h=\frac{Q}{4 \pi T}\left[-0.5772-\log _{e} u+u-\frac{u^{2}}{2 \cdot 2 !}+\frac{u^{3}}{3 \cdot 3 !}-\cdots\right]
$$

where,

$$
u=\frac{1.87 r^{2} S}{T t}
$$

$\mathrm{s}=$ drawdown, in feet,

$\mathrm{Q}=$ discharge, in gpm,

$\mathrm{t}=$ time after pumping started, in days,

$\mathrm{T}=$ coefficient of transmisivity, in gallons per day per foot $(\mathrm{gpd} / \mathrm{ft})$ and

$\mathrm{S}=$ coefficient of storage, dimensionless.

The value of brackets equals $W(u)$. The data for interpolation have logarithm values of $W(u)$ respect to $u$ [6]. For the interpolation of data about ten points are considered. Figure 1 shows the comparison between the

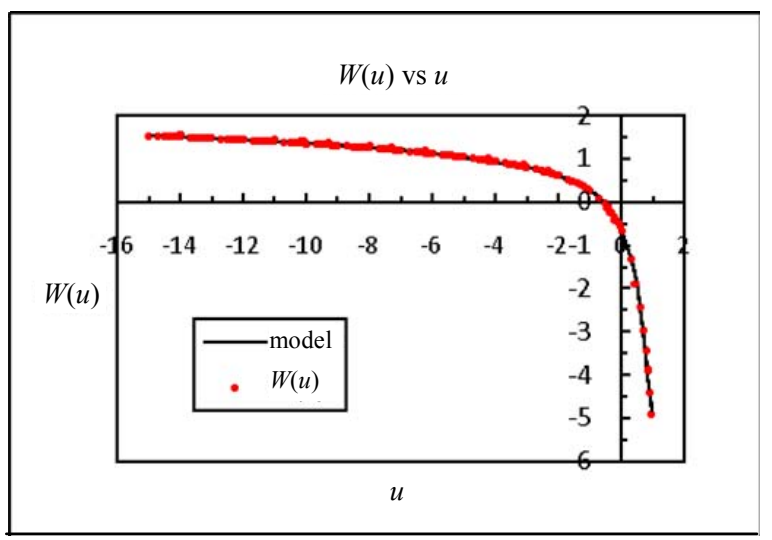

Figure 1. The comparison between the data and the model. 
data and the model for the nine spline curves. Based on the figure there is a suitable and good relationship between those.

\subsection{Example 2}

In this problem about five pairs of data are obtained from the parametric Eq.9 with non-uniform segments.

$$
\left[\begin{array}{l}
x=t \cos \frac{\pi}{12}-2 \sin \sqrt{2 t} \sin \frac{\pi}{12} \\
y=t \sin \frac{\pi}{12}+2 \sin \sqrt{2 t} \cos \frac{\pi}{12}
\end{array}, t \in[0,24] .\right.
$$

About four cubic spline curves are generated according to the formulations of Eqs.3, 4 and 5. Figure 2 shows the relationship between the real values and interpolated ones. As it can be seen, there is a relative expectable fitness between them.

\section{FORMULATION FOR 2-D INTERPOLATION}

The bicubic spline formation that applies hear for two dimensional interpolation and four nodes patch is,

$$
\begin{aligned}
& g_{i j}(x, y)=a_{0}+a_{1} x+a_{2} y+a_{3} x^{2}+a_{4} x y+a_{5} y^{2}+a_{6} x^{3}+ \\
& a_{7} x^{2} y+a_{8} x y^{2}+a_{9} y^{3}
\end{aligned}
$$

or in matrix notation,

$$
\begin{aligned}
& g_{i j}(x, y)=[x][A][y]=\left[\begin{array}{llll}
1 & x & x^{2} & x^{3}
\end{array}\right] \\
& {\left[\begin{array}{cccc}
a_{0} & a_{2} & a_{5} & a_{9} \\
a_{1} & a_{4} & a_{8} & 0 \\
a_{3} & a_{7} & 0 & 0 \\
a_{6} & 0 & 0 & 0
\end{array}\right]\left[\begin{array}{c}
1 \\
y \\
y^{2} \\
y^{3}
\end{array}\right]}
\end{aligned}
$$

If the above equation is written based on $\left(x_{i}, y_{i}\right)$ or the coordinates of the lower left point on each segment, Eq.12

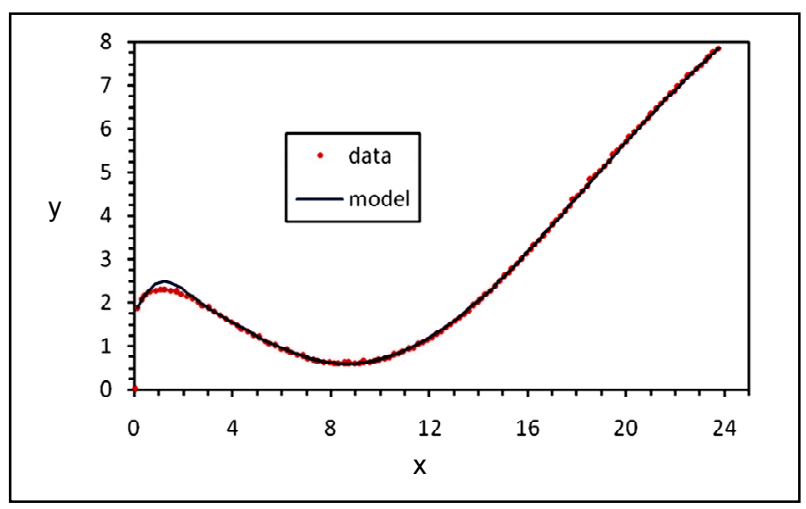

Figure 2. The comparison between the data and the model. obtains. Then, the formulation for determination of the coefficient will be simpler.

$$
\begin{aligned}
s_{i j}(x, y)= & a_{0}+a_{1}\left(x-x_{i}\right)+a_{2}\left(y-y_{j}\right)+a_{3}\left(x-x_{i}\right)^{2} \\
& +a_{4}\left(x-x_{i}\right)\left(y-y_{j}\right)+a_{5}\left(y-y_{j}\right)^{2} \\
& +a_{6}\left(x-x_{i}\right)^{3}+a_{7}\left(x-x_{i}\right)^{2}\left(y-y_{j}\right) \\
& +a_{8}\left(x-x_{j}\right)\left(y-y_{j}\right)^{2}+a_{9}\left(y-y_{j}\right)^{3}
\end{aligned}
$$

or in matrix notation it is,

$$
\begin{aligned}
& s_{i j}(x, y)=\left[\begin{array}{llll}
1 & x & x^{2} & x^{3}
\end{array}\right]\left[\begin{array}{cccc}
1 & -x_{i} & x_{i}^{2} & -x_{i}^{3} \\
0 & 1 & -2 x_{i} & 3 x_{i}^{2} \\
0 & 0 & 1 & -3 x_{i} \\
0 & 0 & 0 & 1
\end{array}\right] \\
& {[A]\left[\begin{array}{cccc}
1 & 0 & 0 & 0 \\
-y_{i} & 1 & 0 & 0 \\
y_{i}^{2} & -2 y_{i} & 1 & 0 \\
-y_{i}^{3} & 3 y_{i}^{2} & -3 y_{i} & 1
\end{array}\right]\left[\begin{array}{c}
1 \\
y \\
y^{2} \\
y^{3}
\end{array}\right]}
\end{aligned}
$$

The above bispline function has ten parameters (unknowns) for each segment or patch. Therefore; ten equations are required for defining them. Four equations satisfy the function values at each corner and six equations satisfying partial derivative in $x$ and $y$ directions for three nodes. Thus there exists the continuity of $C^{1}$ on each of four sides or boundaries of every element. The first derivatives in $x$ and $y$ direction for the Eq.12 are:

$$
\begin{aligned}
\frac{\partial s_{i j}(x, y)}{\partial x}= & {\left[\begin{array}{lll}
1 & 2 x & 3 x^{2}
\end{array}\right]\left[\begin{array}{ccc}
1 & -2 x_{i} & 3 x_{i}^{2} \\
0 & 1 & -3 x_{i} \\
0 & 0 & 1
\end{array}\right] } \\
& {\left[\begin{array}{ccc}
a_{1} & a_{4} & a_{8} \\
a_{3} & a_{7} & 0 \\
a_{6} & 0 & 0
\end{array}\right]\left[\begin{array}{ccc}
1 & 0 & 0 \\
-y_{j} & 1 & 0 \\
y_{j}^{2} & -2 y_{j} & 1
\end{array}\right]\left[\begin{array}{c}
1 \\
y \\
y^{2}
\end{array}\right] . } \\
\frac{\partial s_{i j}(x, y)}{\partial y}= & {\left[\begin{array}{lll}
1 & x & x^{2}
\end{array}\right]\left[\begin{array}{ccc}
1 & -x_{i} & x_{i}^{2} \\
0 & -2 x_{i} \\
0 & 0 & 1
\end{array}\right] } \\
& {\left[\begin{array}{ccc}
a_{2} & a_{5} & a_{9} \\
a_{4} & a_{8} & 0 \\
a_{7} & 0 & 0
\end{array}\right]\left[\begin{array}{ccc}
1 & 0 & 0 \\
-2 y_{j} & 1 & 0 \\
3 y_{j}^{2} & -3 y_{j} & 1
\end{array}\right]\left[\begin{array}{c}
1 \\
2 y \\
3 y^{2}
\end{array}\right] }
\end{aligned}
$$

The coefficients $a_{0}, a_{1}, \ldots, a_{9}$ can be derived explicitly from satisfying the following conditions, 


$$
\begin{gathered}
s_{i j}(i, j)=a_{0}=f_{i j}, \frac{\partial s_{i j}(i, j)}{\partial x}=a_{1}=f_{x, i j}^{\prime}, \\
\frac{\partial s_{i j}(i, j)}{\partial y}=a_{2}=f_{y, i j}^{\prime} \\
\frac{\partial s_{i j}}{\partial x}(i+1, j)=a_{1}+2 h_{i} a_{3}+3 h_{i}^{2} a_{6}=f_{x, i+1, j}^{\prime}, \\
\frac{\partial s_{i j}}{\partial y}(i, j+1)=a_{2}+2 k_{j} a_{5}+3 k_{j}^{2} a_{9}=f_{y, i, j+1}^{\prime}, \\
\frac{\partial s_{i j}}{\partial y}(i+1, j)=a_{2}+h_{i} a_{4}+h_{i}^{2} a_{7}=f_{y, i+1, j}^{\prime}, \\
\frac{\partial s_{i j}}{\partial x}(i, j+1)=a_{1}+k_{j} a_{4}+k_{j}^{2} a_{8}=f_{x, i, j+1}^{\prime}, \\
s_{i j}(i+1, j)=s_{i+1, j}(i+1, j) \Rightarrow \\
a_{0}+h_{i} a_{1}+h_{i}^{2} a_{3}+h_{i}^{3} a_{6}+=f_{i+1, j}, \\
s_{i j}(i, j+1)=s_{i, j+1}(i, j+1) \Rightarrow \\
a_{0}+k_{j} a_{2}+k_{j}^{2} a_{5}+k_{j}^{3} a_{9}+=f_{i, j+1}, \\
s_{i j}(i+1, j+1)=s_{i+1, j+1}(i+1, j+1) \Rightarrow a_{0}+h_{i} a_{1} \\
+k_{j} a_{2}+h_{i}^{3} a_{3}+h_{i} k_{j} a_{4}+k_{j}^{2} a_{5}+h_{i}^{3} a_{6}+h_{i}^{2} k_{j} a_{7} \\
+h_{i} k_{j}^{2} a_{8}+k_{j}^{3} a_{9}=f_{i+1, j+1}
\end{gathered}
$$

The above ten equations give a linear system of equations. If it is solved analytically the governing parameters are obtained as follows,

$$
\begin{aligned}
& a_{3}=-\frac{3}{h_{i}^{2}} a_{0}-\frac{2}{h_{i}} a_{1}+\frac{3}{h_{i}^{2}} f_{i+1, j}-\frac{1}{h_{i}} f_{x, i+1 j}^{\prime} \\
& a_{4}=-\frac{1}{h_{i} k_{j}} a_{0}-\frac{1}{k_{j}} a_{1}-\frac{1}{h_{i}} a_{2}+ \\
& \frac{1}{h_{i} k_{j}}\left(f_{i+1, j}+f_{i, j+1}-f_{i+1, j+1}\right)+\frac{1}{k_{j}} f_{x, i j+1}^{\prime}, \\
& a_{5}=-\frac{3}{k_{j}^{2}} a_{0}-\frac{2}{k_{j}} a_{2}+\frac{3}{k_{j}^{2}} f_{i, j+1}-\frac{1}{k_{j}} f_{y, i j+1}^{\prime}, \\
& a_{6}=\frac{2}{h_{i}^{3}} a_{0}+\frac{1}{h_{i}^{2}} a_{1}-\frac{2}{h_{i}^{3}} f_{i+1, j}+\frac{1}{h_{i}^{2}} f_{x, i+1 j}^{\prime}, \\
& a_{7}=-\frac{1}{h_{i}^{2} k_{j}} a_{0}+\frac{1}{h_{i} k_{j}} a_{1}-\frac{1}{h_{i}^{2} k_{j}} \\
& \left(f_{i+1, j}+f_{i, j+1}-f_{i+1, j+1}\right)-\frac{1}{h_{i} k_{j}} f_{x, i j+1}^{\prime}, \\
& a_{8}=\frac{1}{h_{i} k_{j}^{2}} a_{0}+\frac{1}{h_{i} k_{j}} a_{2}-\frac{1}{h_{i} k_{j}^{2}}\left(f_{i+1, j}+\right. \\
& \left.f_{i, j+1}-f_{i+1, j+1}\right)-\frac{1}{h_{i} k_{j}} f_{y, i+1 j}^{\prime}
\end{aligned}
$$

$$
a_{9}=\frac{2}{k_{j}^{3}} a_{0}+\frac{1}{k_{j}^{2}} a_{2}-\frac{2}{k_{j}^{3}} f_{i, j+1}+\frac{1}{k_{j}^{2}} f_{y, i j+1}^{\prime}
$$

where $h_{i}=x_{i+1}-x_{i} \quad, \quad k_{j}=y_{j+1}-y_{j}$,

$$
f_{x, i j+1}^{\prime}=\frac{\partial f}{\partial x}(i, j+1) \quad, \quad f_{y, i+1 j}^{\prime}=\frac{\partial f}{\partial y}(i+1, j) .
$$

\section{PROBLEM 3}

For testing the above formulation several problems were considered. Here only one of them is presented. The data for this problem are obtained from the Eq.32.

$$
z(x, y)=4 e^{-\left(0.0867 x^{2}+0.5 y^{2}\right)}, \quad x, y \in[0,6] \times[0,3]
$$

The domain of problem consists of 9 elements and 16 nodes. The elements are uniform where $h_{i}=2$ and $k_{i}=1$. The above formulations were applied to to the governing data. Figure 3 shows the interpolation curves along $x=0, x=2, y=0$ and $y=2$. As it can be seen the 2-D model presents a close relationship to the relating set of data.

\section{THE RESULTS AND CONCLUSIONS}

In this research two models for 1-D and 2-D interpolations were presented. The models are easy and straightforward to handle. Therefore; it can be applied for problems having huge data. It has the advantage of less computational efforts respect to the cubic spline, Bezier and B-spline methods especially for 2-D problems. The continuity for each segment is $C^{1}$ therefore; this is sufficient for many problems such as calculation of surfaces and volumes of nonuniform bodies. It is recommended to compare the 2-D model with Bezier and B-spine methods respect to the fitness and accuracy.

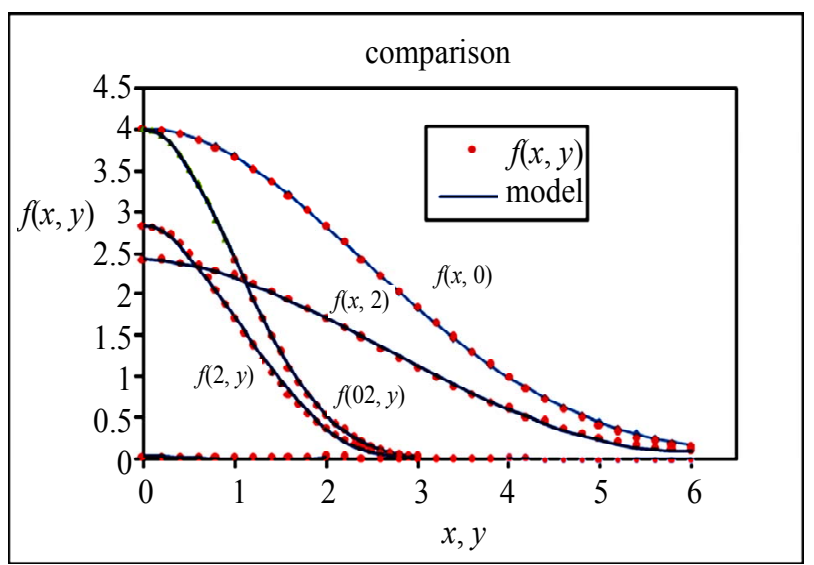

Figure 3. The comparison between the data and 2-D model. 


\section{REFERENCES}

[1] Salmon, D. (2006) Curves and surfaces for computer graphics. Springer, New York.

[2] Saxena, A. and Sahay, B. (2005) Computer aided engineering design. Springer, Anamaya Publisher, New Delhi.

[3] Conte, S.D. and de Boor, C. (1981) Elementary numeri-

cal analysis, an algorithm approach. Kin Keong Printing Co. Pte. Ltd., Singapore.

[4] Burden, R. and Fairs, D. (1989) Numerical analysis. PWS-Kent Publishing Company, Boston.

[5] March, D. (2005) Applied geometry for compute graphics and CAD. 2nd Edition, Springer-Verlag, New York.

[6] Todd, D.K. (1959) Groundwater hydrology. John Wily \& Sons, Inc., New York. 